\title{
ELASTIC CONSTANTS OF DENSE CRYSTALLINE PHASES OF TWO-DIMENSIONAL HARD CYCLIC HEPTAMERS
}

\author{
K. W. WOJCIECHOWSKI, K. V. TRETIAKOV \\ Institute of Molecular Physics, Polish Academy of Sciences \\ Smohichowskiego 17/19, 60-179 Poznań, Poland \\ kww@man.poznan.pl
}

\begin{abstract}
Two-dimensional system of hard cyclic heptamers is studied by Monte Carlo simulations. Isotherms of various crystalline structures formed by the heptamers are obtained. Melting as well as structural phase transitions between crystalline structures are localized. Above melting a rotational phase is observed of hexagonal lattice and isotropic distribution of molecular orientations. With increasing density the coupling between the orientational and rotational degrees of freedom leads to appearance of clearly anisotropic patterns of the atomic density distribution around the lattice sites. These patterns exhibit 6-fold symmetry. At high densities the heptamers form crystalline structures of rectangular lattice and without molecular rotation. Elastic constants of the dense crystalline structures are computed by using an algorithm based on the strain-fluctuation method. It is shown that the densest known crystalline structures of the heptamers exhibit negative Poisson's ratios near close packing.
\end{abstract}

\section{I NTRODUCTION}

The planar hard cyclic heptamer molecule, for simplicity further referred to as heptamer, consists of seven equidiameter hard discs-"atoms"1 ${ }^{11}$ whose centres form a perfect.heptagon of sides equal to the diameter of the discs. Although the heptamer "molecule", with its purely geometrical interactions, does not exist in nature, there are a few reasons for which the thermodynamic and structural properties of systems composed of such molecules are worth to study. These reasons are discussed below.

The heptamer is an example of a hard body. Hard body systems are, in general, important reference systems in the theory of fluids [1,2]. Systems of hard molecules can also model other condensed matter phases. Using molecules of various shapes one can obtain, e.g., liquid crystalline phases [2], various crystalline phases, including orientationally disordered phases [3], and even thermodynamically stable, aperiodic solid phases (degenerate crystals) [4]. Models with hard interactions are attractive (both from the point of view of the theory and computer simulations) for the simplicity of their phase diagrams. Purely geometric interactions imply that for such models the whole phase diagram can be obtained by simple scaling of a single isotherm. This simple scaling (by temperature) can be also applied to some important thermodynamic quantities, like pressure or elastic constants, describing the hard body systems.

${ }^{1}$ The interaction potential of two hard discs is zero if the distance, $r$, between the centres of the discs is not less than the disc diameter, $\sigma$. Otherwise, if the discs overlap $(r<\sigma)$, the interaction potential is infinite. 
In studies of hard-body systems, particular attention has been paid to the convex bodies [2] because various rigorous results arc known for them [5-7] and using convex bodies one can speed up simulations in some thermodynamic ensembles. Although the heptamer is not a convex molecule, it belongs to so called star-shaped bodies ${ }^{2}$. The star-shaped bodies, being as convenient as the convex bodies in certain computer simulations [8], can model broader class of molecules than the convex bodies. At present, however, relatively few results are available for the hard starshaped bodies. In this context the study of hard heptamer system is useful as it enlarges the amount of available data on non-convex hard star-shaped bodies.

The heptamer is one of the simplest examples of a molecule exhibiting the 7-fold symmetry axis. This axis is the next lowest axis, after the 5-fold one, forbidden in periodic crystalline phases. It is interesting, in general, what kinds of dense structures can be obtained from highly symmetric molecules of symmetries forbidden in crystals. In contrast to various studies of model systems containing particles of pentagonal-like shape [9-11], the present authors are not aware of any investigations of planar particles of heptagonal-like shape.

Another interesting problem is related to dense structures of heptamers. The close packed structure observed in simulations of hard cyclic pentamers (which are composed of five discsatoms with centers placed at vertices of a perfect pentagon of sides equal to the disc diameters) is formed of pentamer rows showing "anti-parallel" orientations [10, 11]. It can be shown that the heptamers can form two close packed structures of this kind and these structures have almost the same density. It is interesting if this quasi-degeneracy shows any influence on the phase diagram and properties of the system. Such studies can be useful for better understanding thermodynamic stability of solid phases.

Being an anisotropic and non-convex molecule, the heptamer is also an attractive candidate for testing some computational methods. In particular, one can check how a simple method of calculating the elastic properties of solid phases by computer simulation [12] will work for an anisotropic system. Moreover, as such calculations require generating very long cycles of random numbers, they can be also used as practical efficiency tests of some random number generators [13-15],

Last but not least, it is worth to mention that even a study of very artificial systems can be a source of important information concerning real systems. (The best known example is the Ising model.) Some years ago, it has been pointed out that certain systems of anisotropic (non-convex) hard-bodies can exhibit anomalous Poisson's ratio at high densities [16], It is interesting if such a counterintuitive behaviour can be observed in the heptamer system. It should be stressed here that systems of negative Poisson's ratio are not just theoretical curiosities but can find a lot of interesting applications. They have been manufactured more than a decade ago [17] and since that time they have been a subject of very intensive studies [18],

The main aim of this paper is to determine the elastic properties of the densest phases of heptamers by applying the strain-fluctuation method [12], This study extends the amount of 2

Any point of a star-shaped body can be connected with a point, called star-point, by a straight-line segment contained in the body. Each point of a convex body is the star-point. 
available data concerning systems consisted of anisotropic molecules interacting through strongly anharmonic potentials. Such data are useful to construct various theoretical approximations and necessary to test such approximations. As a by-product we obtain some information on the equation of state and structural properties of the heptamers.

The paper is organized as follows. The Section 2 concerns the equation of state (EOS) and structural properties of the heptamer system. In Section 3, the elastic constants of dense crystalline structures of the heptamers are discussed. The Section 4 contains a summary and conclusions.

\section{THE EOS OF THE HEPTAMER SYSTEM}

Usually, the structure of the ground state of a system determines the structure and the properties of its lowest-temperature phase. So, simulating a low-temperature crystalline phase of a system, it is natural to start simulations from the ground state configuration. In the case of hardbody systems the latter one corresponds to the system structure at close packing. When the ground state (the close packed structure) of a system is not known before simulations, one may try to find it by using the simulations. For smooth interaction potentials the most natural way to achieve this task is to start from a high-temperature (disordered) state and then decrease the temperature slowly enough to allow the system for annealing possible defects. The final state (usually well ordered) reached in such a process can be considered as stable (or, at least, meta-stable) at the lowest temperatures. For hard-body systems the corresponding process of searching for the close packed structure requires increasing the pressure to temperature ratio.

In principle, one could expect that within such a computational experiment the complete EOS would be determined for the system studied. In fact, however, there is a possibility that some metastable phases are obtained, what should modify interpretation of obtained results. For this reason it is meaningful to perform the next computational experiment by continuing the simulations in the opposite direction, i.e. with decreasing pressure to temperature ratio. Obviously, obtaining the same results in both experiments is a necessary condition to expect that the obtained data represent the EOS of the system. However, one should be aware that this is not a sufficient condition, in general. The studies of the heptamer system, described below, clearly illustrate such a possibility.

The structure of the densest phase of the heptamers was not known before the simulations. Thus, constant pressure Monte Carlo simulations (of $N=780$ molecules) in a box of variable shape,see e.g. [19], were initiated at veiy low value of the dimensionless pressure, $p^{*}=p \sigma^{2} / k T=0.1$, where the thermodynamically stable phase is fluid. In the fluid phase the system was both translationally and orientationally disordered. After equilibration, the dimensionless pressure was slowly increased in subsequent runs what resulted in decreasing the system volume ${ }^{3}$. Freezing of the fluid was observed around $p^{*} \approx 1.0$, see Fig. 1 . Above the freezing transition the fact that the shape of the simulation box was relaxed became important because the system could "choose" the equilibrium structure. Hexagonal lattice of the mean positions of the molecular mass centres was

As the system is planar the volume means the area of the system. In this paper a dimensionless volume, being the ratio of the system area to its area at the closest known packing, is used. 


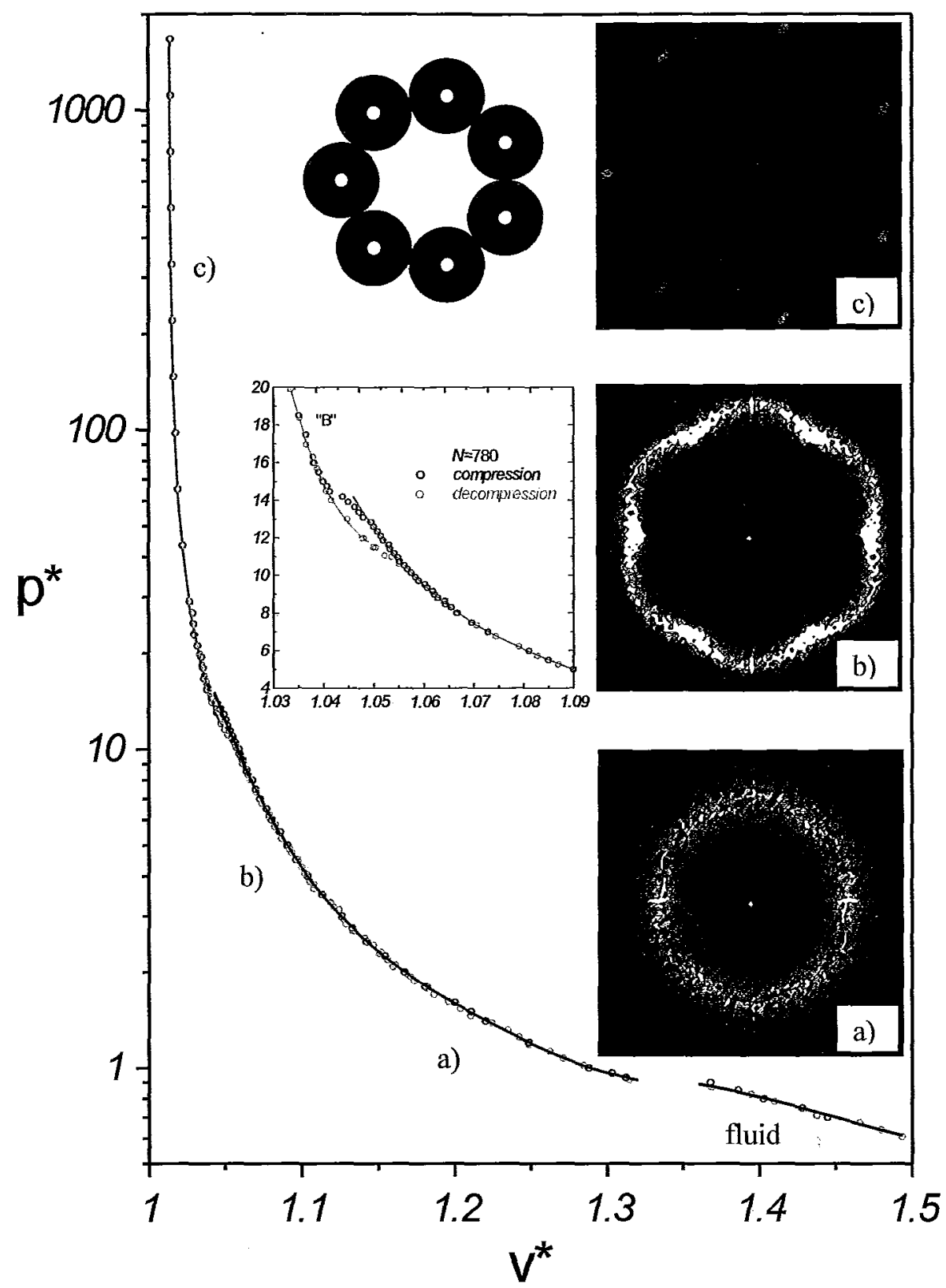

Fig. 1. The EOS of the heptamers. The dimensionless volume $v^{*} i s$ defined as the ratio of the volume of the system to the volume of the close packed structure denoted by J2 (see Fig. 4c) which is the densest known structure of the heptamers. The heptamer molecule is shown in the left upper comer. The insert below it presents the enlarged transition region between the hexagonal crystalline phase with molecular rotation (rotational phase) and a crystalline structure without molecular rotation (the B-structure), see Fig. 4b. The inserts on the right show the atomic probability density around a crystalline lattice site a) in the low-density rotational phase $\left.\left(p^{*}=2\right), \mathrm{b}\right)$ in the high-density rotational phase $\left(p^{*}=6\right)$, and c) in the B-structure $\left(p^{*}=20\right)$, respectively 


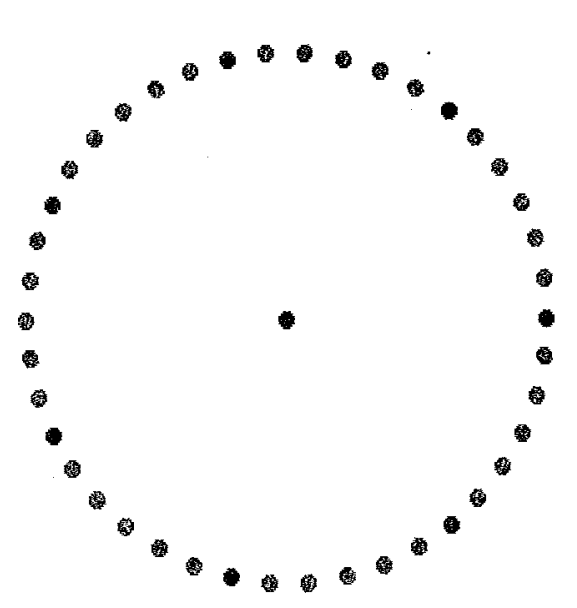

a)

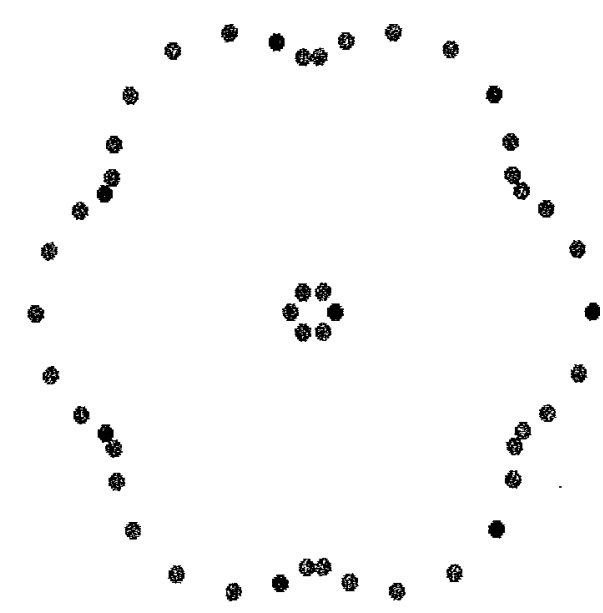

b)

Fig. 2. An example explaining the influence of translational-rotational (TR) coupling on the symmetry of the "atomic" patterns formed by heptamers around the lattice sites: a) a highly symmetric pattern formed without any TR coupling, b) a pattern of 6-fold symmetry obtained at a strong TR coupling. The red dots represent centres of the discs and the molecular centre at "zero" orientation, the green dots are obtained by applying to this molecule rotations by $k \pi / 6(k=1, \ldots, 5)$ with respect to the centre of the coordinate system

formed. However, the molecular orientations remained disordered, the molecules were almost freely rotating, and the "atomic" density distribution was seen as ring-like patterns around the lattice sites; an example is shown in Fig. la. Above $p^{*} \approx 2.8$, although the molecules were still able to rotate, some structure of the 6 -fold symmetry became visible in the atomic distribution around the lattice sites, see Fig. 1b. This indicated a strong coupling between orientational and translational degrees of freedom in the system. The origin of the 6-fold symmetry of the found pattern is illustrated in Fig. 2. Such behaviour was earlier observed in the planar hard cyclic pentamer system [10],

The hexagonal structure remained qualitatively unchanged (but some quantitative changes were observed: the distances between the lattice sites were decreasing, the pattern around the lattice sites was becoming sharper, and the molecular rotation was slower at higher densities) when $p^{*}$ was increased up to $p^{*} \approx 13.5$. Above this value the symmetry of the lattice of the molecular centres has been spontaneously reduced and the molecular rotation froze. The unit cell consisted of two molecules and the atomic pattern around the lattice sites showed clearly 7 -fold symmetry, see Fig. 1c. No qualitative changes have been observed in the system when $p^{*}$ was further increased up to the value $p^{*}=10^{7}$ (which can be seen as infinity) and the system tended to the close packed structure marked in Fig. 3 as the B-structure.

To check if the system would behave in the same way when $p^{*}$ is decreased, the second experiment was performed, starting from the final state of the first experiment. The results were the same except some hysteresis near the structural (first order) phase transition in the solid 


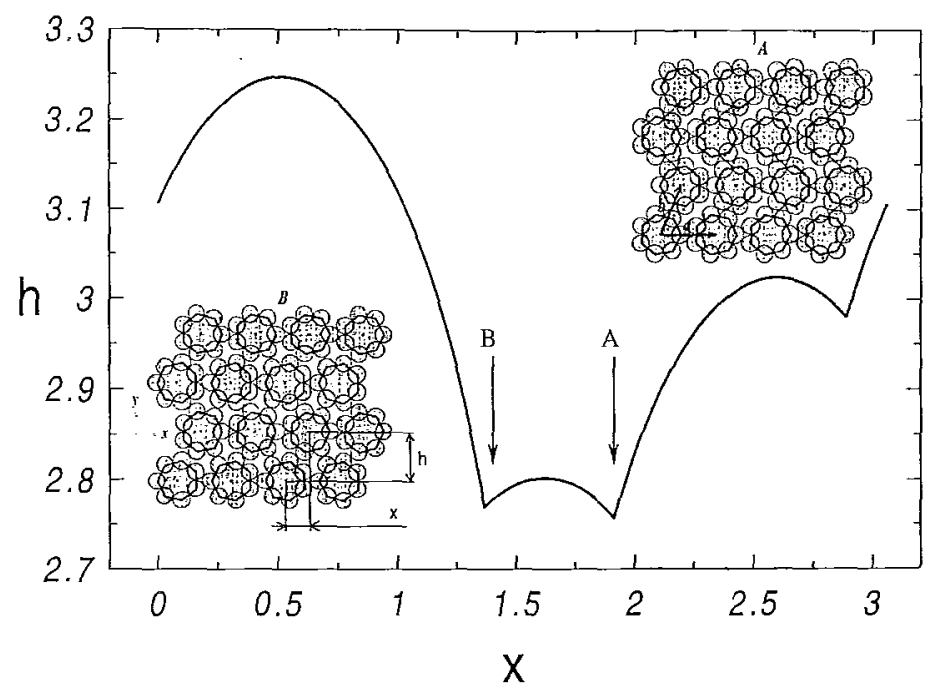

Fig. 3. The minimum distance of neighbouring "anti-parallel" rows of the heptamers (all molecules in a row have the same orientation, zero or $\pi$ ) versus the minimum distance of perpendicular projections of the centres of two heptamers representing different rows on the row direction
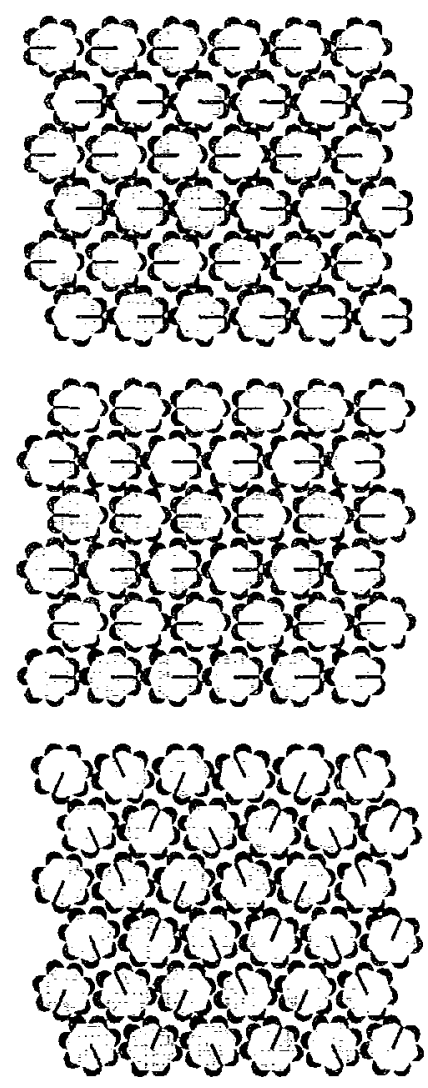

a)

Fig. 4. Three close packed structures of the heptamers for which the elastic constants were studied in this paper: a) the Astructure of rectangular lattice and two molecules per unit cell, b) the B-structure of rectangular lattice and two molecules per unit cell, c) the J2-structure of rectangular lattice and four molecules per unit cell. The line segments with one end in the

b) molecular centre represent the molecular orientation angle (with respect to the $\mathrm{x}$-axis) multiplied by 7 . It can be seen that the lattice of the heptamer mass centres of the B-structure is much closer to the hexagonal lattice than the corresponding lattice of the A-structure. The $\mathrm{J} 2$ structure is the densest of known structures formed by the heptamers. 
phase, see the insert in Fig. 1. The transition from the B-structure to the hexagonal crystal with rotation was observed at $p^{*} \approx 10.5$ what is about 30 percent less than when the system was compressed. Some small hysteresis was also observed near melting.

At a first look the described above simulation results might suggest that the problem of the heptamer phase diagram has been solved. Namely, one might expect that only thermodynamically stable phases have been observed in the whole pressure range. However, a closer inspection of the Fig. 3, clearly indicates that the crystalline structure, denoted by A, which is denser at close packing than the B-structure found in the simulations. It is worth to notice that the lattice of the B-structure is closer to the hexagonal lattice than the lattice of the A-structure.

The packing argument implies that the B-structure observed in the simulation cannot be thermodynamically stable in the whole density range where the molecular rotation is frozen. The simplest scenario consistent with the described simulations and the close packing argument would require presence of a phase transition between the structures A and B at very high densities. Such

Fig. 5. The isotherms of the A, B, and J2 crystalline structures of the heptamers

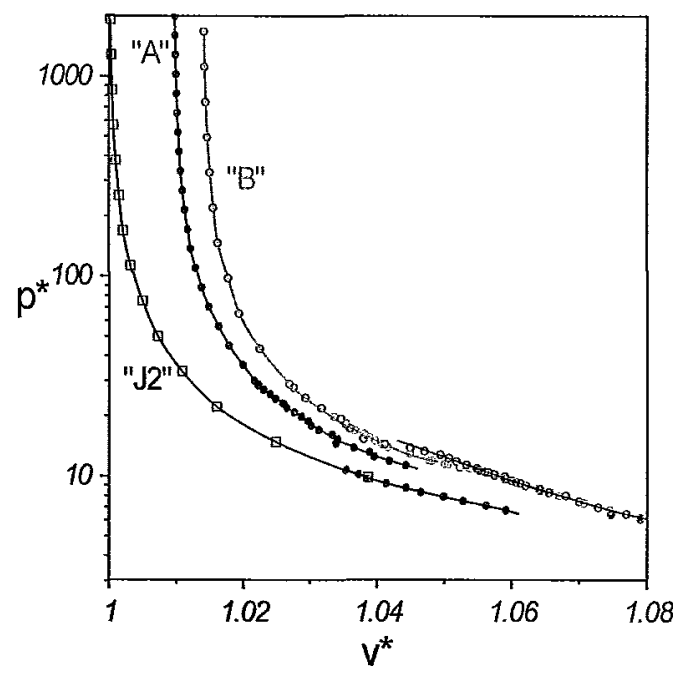

a transition might be revealed at decompression of the A-structure. Further studies were then necessary.

The next simulation experiment was started from the A-structure at the dimensionless pressure $p^{*}=2000$. In subsequent runs the pressure was slowly reduced. No qualitative change of the Astructure was observed down to $p^{*} \approx 11$ where the system rapidly decreased the relative volume and "jumped" to a new structure shown in Fig. 4c. In this paper the latter structure will be referred to as the $\mathrm{J} 2$-structure. The $\mathrm{J} 2$-structure was observed until a transition to the hexagonal lattice with hindered rotation of the molecules has occurred at $p^{*} \approx 6.5$. Below this pressure the same scenario was found as in the first experiment when the B-structure was decompressed.

It should be stressed that the J2-structure is the densest of the known packings of the heptamers. When compressed from the state of pressure just above the spontaneous transformation to 
the hexagonal lattice with rotation, the $\mathrm{J} 2$-structure remained qualitatively unchanged up to $p^{*}=10^{7}$. It should be noticed, however, that also the A-structure remained qualitatively unchanged when compressed up to $p^{*}=10^{7}$ from the state of pressure just above its spontaneous transformation to the $\mathbf{J} 2$-structure. This proves that the free energy barriers between various crystalline structures of the pentamers are very high at high densities. The isotherms of the structures $\mathrm{A}, \mathrm{B}$, and $\mathrm{J} 2$ are shown in Fig. 5 .

The above results indicate that the heptamer fluid freezes into the hexagonal crystalline phase in which molecules can rotate. With increasing density the rotation of molecules changes from almost free one to strongly hindered one and, finally, a structural transition occurs to a phase with frozen rotation. It is not known at present what are the stability ranges, if any, of the structures $\mathrm{A}, \mathrm{B}$, and J2. Solution of this problem requires a comparison of the free energies of these structures. However, this will not be the subject of the present study because the obtained results prove that all these structures are at least metastable in a certain high-density region. This is sufficient to enable one for computing their elastic properties in the (meta-)stability range by using the strain-fluctuation method what is the subject of the following section.

\section{ELASTIC PROPERTIES OF THE HEPTAMER SYSTEM}

Computations of elastic properties of the heptamer crystalline structures were performed by the strain-fluctuations method [12, 20], We will not describe this method here, restricting ourselves to the basic definitions only.

The (variable) periodic box is such that it can be described by the symmetric box matrix, $h_{i j}$, the columns of which are formed by the components of the edge-vectors of the box. The (Lagrange) strain tensor, $\varepsilon_{i j}$, can be then written as [20]

$$
\underline{\varepsilon}=\frac{\left(\underline{h}_{0}^{-1} h \underline{h} \underline{h}_{0}^{-1}-L\right)}{2}
$$

where the zero index denotes the reference state. In the constant pressure simulations, it is convenient to define the reference state as the equilibrium state at the simulation pressure. The components of the tensor of the elastic compliances [12] can be then expressed by fluctuations of the components of the strain tensor as follows

$$
\frac{\langle V\rangle_{p}}{k T}<\varepsilon_{i j} \varepsilon_{k l}>=\bar{S}_{i j k l}
$$

where the meaning of the averages is discussed in [12] and volume is calculated as $V=\operatorname{det}(h)$. For two-dimensional hard-body systems it is convenient to use dimensionless elastic compliances:

$$
S_{i j k l}=\frac{\bar{S}_{i j k l} k T}{\sigma^{2}}
$$


Table I. The equation of state and the dimensionless elastic compliances of the B-structure for various numbers, $N$, of the heptamers in the system.

The Voigt notation is used here and in the following tables and figures

\begin{tabular}{|c|c|c|c|c|c|c|c|c|}
\hline$N$ & $p^{*}$ & $v^{*}$ & $S_{11}$ & $S_{12}$ & $S_{16}$ & $S_{22}$ & $S_{26}$ & $S_{66}$ \\
\hline 36 & 15 & $1.0404(6)$ & $0.00210(7)$ & $-0.00059(2)$ & $0.00004(4)$ & $0.00086(2)$ & $-0.00002(2)$ & $0.00126(6)$ \\
\hline 56 & 15 & $1.0402(5)$ & $0.00218(7)$ & $-0.00059(1)$ & $0.00000(3)$ & $0.00085(1)$ & $0.00000(1)$ & $0.00122(3)$ \\
\hline 224 & 15 & $1.0401(3)$ & $0.00214(4)$ & $-0.00061(1)$ & $0.00000(3)$ & $0.00085(\mathrm{l})$ & $0.00002(1)$ & $0.00117(3)$ \\
\hline 36 & 20 & $1.03326(5)$ & $0.00116(4)$ & $-0.000271(17)$ & $-0.000007(14)$ & $0.000437(7)$ & $0.000002(8)$ & $0.00061(2)$ \\
\hline 56 & 20 & $1.03321(2)$ & $0.00111(2)$ & $-0.000260(10)$ & $0.000010(11)$ & $0.000425(6)$ & $-0.000002(3)$ & $0.00058(1)$ \\
\hline 36 & 30 & $1.02640(2)$ & $0.000409(9)$ & $-0.000075(3)$ & $-0.000001(3)$ & $0.000172(4)$ & $0.000002(4)$ & $0.000238(8)$ \\
\hline 56 & 30 & $1.02638(1)$ & $0.000399(7)$ & $-0.000077(2)$ & $0.000001(3)$ & $0.000176(3)$ & $-0.000003(2)$ & $0.000232(4)$ \\
\hline 36 & 50 & $1.02126(4)$ & $0.000128(4)$ & $-0.000021(2)$ & $0.000002(2)$ & $0.000061(2)$ & $0.0000003(5)$ & $0.000080(2)$ \\
\hline 56 & 50 & $1.02123(2)$ & $0.000125(2)$ & $-0.000021(2)$ & $-0.000001(2)$ & $0.000060(1)$ & $-0.000000(4)$ & $0.000080(2)$ \\
\hline 224 & 50 & $1.02121(1)$ & $0.000130(3)$ & $-0.000020(1)$ & $0.000002(1)$ & $0.000059(1)$ & $0.000000(1)$ & $0.000077(1)$ \\
\hline
\end{tabular}


Table II. The equation of state and the dimensionless elastic compliances of the A-structure of the heptamers $(N=36)$

\begin{tabular}{|c|c|c|c|c|c|c|c|}
\hline$p^{*}$ & $v^{*}$ & $S_{11}$ & $S_{22}$ & $S_{12}$ & $S_{66}$ & $S_{62}$ & $S_{16}$ \\
\hline 1 & 2 & 3 & 4 & 5 & 6 & 7 & 8 \\
\hline 1000 & $1.009992(6)$ & $0.000000152(18)$ & $0.000000132(19)$ & $0.000000011(16)$ & $0.00000021(3)$ & $0.000000004(13)$ & $-0.000000023(7)$ \\
\hline 860 & $1.010043(2)$ & $0.000000192(7)$ & $0.000000207(6)$ & $0.000000050(06)$ & $0.000000352(10)$ & $0.0000000022(5)$ & $0.000000018(10)$ \\
\hline 739.6 & $1.010113(2)$ & $0.000000258(8)$ & $0.000000291(12)$ & $0.000000050(03)$ & $0.000000465(8)$ & $0.000000001(14)$ & $-0.000000006(9)$ \\
\hline 636.056 & $1.010194(2)$ & $0.00000036(2)$ & $0.000000375(8)$ & $0.000000089(12)$ & $0.00000063(3)$ & $-0.000000018(16)$ & $-0.000000019(8)$ \\
\hline 547.008 & $1.010285(2)$ & $0.000000479(9)$ & $0.000000491(17)$ & $0.000000087(05)$ & $0.00000087(4)$ & $0.00000003(2)$ & $0.000000000(2)$ \\
\hline 470.427 & $1.010396(2)$ & $0.00000070(2)$ & $0.00000068(2)$ & $0.000000136(13)$ & $0.00000120(3)$ & $-0.00000002(3)$ & $0.000000005(17)$ \\
\hline 404.567 & $1.010517(3)$ & $0.00000092(3)$ & $0.000000932(19)$ & $0.00000020(2)$ & $0.00000169(5)$ & $0.00000002(4)$ & $0.00000001(5)$ \\
\hline 347.928 & $1.010659(2)$ & $0.00000116(5)$ & $0.000001266(16)$ & $0.000000230(18)$ & $0.00000215(9)$ & $-0.00000006(4)$ & $-0.00000005(5)$ \\
\hline 299.218 & $1.010830(3)$ & $0.00000157(4)$ & $0.00000170(7)$ & $0.00000027(7)$ & $0.00000275(13)$ & $0.00000004(6)$ & $-0.00000002(5)$ \\
\hline 257.327 & $1.011022(3)$ & $0.00000203(9)$ & $0.00000251(3)$ & $0.00000051(5)$ & $0.00000402(17)$ & $0.00000005(5)$ & $-0.00000021(11)$ \\
\hline 221.302 & $1.011264(5)$ & $0.00000289(7)$ & $0.00000310(3)$ & $0.00000046(6)$ & $0.0000050(3)$ & $0.00000008(9)$ & $-0.00000003(7)$ \\
\hline 190.319 & $1.011537(7)$ & $0.00000411(13)$ & $0.00000413(8)$ & $0.00000083(11)$ & $0.0000074(3)$ & $0.00000007(15)$ & $-0.00000019(5)$ \\
\hline 163.675 & $1.011840(10)$ & $0.00000540(3)$ & $0.0000054(3)$ & $0.00000094(19)$ & $0.0000093(3)$ & $0.00000021(16)$ & $-0.00000001(17)$ \\
\hline 140.76 & $1.012213(16)$ & $0.0000075(3)$ & $0.00000777(19)$ & $0.00000177(14)$ & $0.0000128(3)$ & $-0.0000002(4)$ & $0.0000001(3)$ \\
\hline 121.054 & $1.012668(18)$ & $0.0000102(4)$ & $0.0000112(4)$ & $0.00000220(11)$ & $0.0000194(4)$ & $-0.0000001(3)$ & $0.0000002(4)$ \\
\hline 104.106 & $1.013112(12)$ & $0.00001266(19)$ & $0.0000140(6)$ & $0.00000275(13)$ & $0.0000231(7)$ & $0.0000004(5)$ & $-0.0000003(3)$ \\
\hline 89.531 & $1.013677(11)$ & $0.0000174(5)$ & $0.0000186(6)$ & $0.0000033(3)$ & $0.0000302(15)$ & $-0.0000006(7)$ & $0.0000008(4)$ \\
\hline 76.997 & $1.01435(3)$ & $0.0000253(9)$ & $0.0000254(10)$ & $0.0000057(9)$ & $0.000042(2)$ & $0.0000002(10)$ & $-0.0000001(5)$ \\
\hline 66.217 & $1.015101(18)$ & $0.0000328(11)$ & $0.0000347(8)$ & $0.0000065(5)$ & $0.0000609(16)$ & $0.0000021(10)$ & $0.0000002(12)$ \\
\hline 56.947 & $1.01607(2)$ & $0.0000436(15)$ & $0.0000462(19)$ & $0.0000084(6)$ & $0.000094(7)$ & $0.000001(3)$ & $0.0000010(18)$ \\
\hline
\end{tabular}




\begin{tabular}{cccccccc}
\hline 1 & 2 & 3 & 4 & 5 & 6 & 7 & 8 \\
\hline 48.974 & $1.01712(4)$ & $0.0000593(19)$ & $0.0000666(11)$ & $0.0000118(12)$ & $0.000131(5)$ & $-0.0000026(18)$ & $-0.0000010(19)$ \\
42.118 & $1.018312(16)$ & $0.000083(3)$ & $0.000089(2)$ & $0.000015(2)$ & $0.000159(7)$ & $-0.0000062(9)$ & $-0.000002(2)$ \\
36.221 & $1.019776(10)$ & $0.000103(7)$ & $0.000123(2)$ & $0.000021(4)$ & $0.000235(5)$ & $0.000005(7)$ & $0.00004(5)$ \\
31.15 & $1.02151(4)$ & $0.000158(9)$ & $0.000154(9)$ & $0.000030(4)$ & $0.000306(13)$ & $0.000007(7)$ & $0.000014(6)$ \\
26.789 & $1.02356(8)$ & $0.000224(14)$ & $0.000242(3)$ & $0.000051(7)$ & $0.00048(3)$ & $0.0000003(19)$ & $-0.000016(7)$ \\
23.039 & $1.02583(8)$ & $0.000282(10)$ & $0.000305(6)$ & $0.000046(12)$ & $0.00068(3)$ & $-0.000006(17)$ & $0.000005(15)$ \\
19.813 & $1.02876(2)$ & $0.000399(8)$ & $0.00043(2)$ & $0.000076(7)$ & $0.00106(6)$ & $-0.000039(15)$ & $-0.000031(14)$ \\
17.04 & $1.0263(2)$ & $0.0020(12)$ & $0.00057(3)$ & $-0.00013(17)$ & $0.00092(15)$ & $0.000004(13)$ & $0.000019(12)$ \\
14.654 & $1.02550(11)$ & $0.00096(4)$ & $0.000669(18)$ & $0.00003(3)$ & $0.00080(3)$ & $-0.000023(12)$ & $-0.000033(16)$ \\
12.602 & $1.03007(12)$ & $0.00134(9)$ & $0.00097(2)$ & $0.00004(2)$ & $0.00122(3)$ & $-0.00002(2)$ & $-0.00003(4)$ \\
10.838 & $1.03500(19)$ & $0.00207(5)$ & $0.00118(5)$ & $0.00007(3)$ & $0.00156(6)$ & $-0.00001(2)$ & $-0.00001(6)$ \\
9.321 & $1.0416(2)$ & $0.00293(11)$ & $0.00172(3)$ & $0.00011(2)$ & $0.00244(5)$ & $0.00005(5)$ & $-0.00012(8)$ \\
8.016 & $1.0495(3)$ & $0.0043(2)$ & $0.00227(5)$ & $-0.00013(6)$ & $0.00376(11)$ & $0.00003(10)$ & $-0.0001(2)$ \\
6.894 & $1.0739(3)$ & $0.00712(33)$ & $0.00661(39)$ & $-0.00347(46)$ & $0.00526(37)$ & $0.00008(20)$ & $-0.00014(21)$ \\
5.929 & $1.08145(18)$ & $0.00808(25)$ & $0.00739(45)$ & $-0.00357(25)$ & $0.00623(17)$ & $0.00047(21)$ & $-0.00063(12)$ \\
5.099 & $1.0901(2)$ & $0.00897(18)$ & $0.00976(41)$ & $-0.00414(7)$ & $0.00672(16)$ & $-0.00028(11)$ & $0.00057(16)$ \\
4.385 & $1.0989(3)$ & $0.01128(42)$ & $0.01057(50)$ & $-0.00471(34)$ & $0.00781(11)$ & $0.00076(36)$ & $-0.00043(18)$ \\
3.771 & $1.1097(3)$ & $0.01426(58)$ & $0.01486(41)$ & $-0.00587(23)$ & $0.00978(40)$ & $-0.00019(21)$ & $0.00029(29)$ \\
3.243 & $1.1203(3)$ & $0.01814(145)$ & $0.01790(44)$ & $-0.00728(50)$ & $0.01263(57)$ & $-0.00022(36)$ & $-0.00024(43)$ \\
2.789 & $1.1334(2)$ & $0.02167(75)$ & $0.02243(87)$ & $-0.00821(59)$ & $0.01528(31)$ & $-0.00030(60)$ & $0.00051(58)$ \\
\hline
\end{tabular}


Table III. The equation of state and the dimensionless elastic compliances of the B-structure of the heptamers $(\mathrm{N}=36)$

\begin{tabular}{|c|c|c|c|c|c|c|c|}
\hline$p^{*}$ & $\mathrm{v}^{*}$ & $S_{11}$ & $S_{22}$ & $S_{12}$ & $S_{66}$ & $S_{62}$ & $S_{16}$ \\
\hline 1 & 2 & 3 & 4 & 5 & 6 & 7 & 8 \\
\hline 1000 & $1.0142428(13)$ & $0.000000282(13)$ & $0.000000148(8)$ & $-0.000000038(4)$ & $0.000000193(9)$ & $0.000000005(3)$ & $0.000000010(6)$ \\
\hline 860 & $1.0143034(16)$ & $0.000000385(23)$ & $0.000000200(3)$ & $-0.000000059(2)$ & $0.000000260(6)$ & $-0.000000005(7)$ & $0.000000016(14)$ \\
\hline 739.6 & $1.0143741(16)$ & $0.000000526(13)$ & $0.000000260(6)$ & $-0.000000073(8)$ & $0.000000345(9)$ & $-0.000000010(14)$ & $-0.000000000(15)$ \\
\hline 636.056 & $1.014455(2)$ & $0.00000070(6)$ & $0.000000362(16)$ & $-0.000000101(9)$ & $0.00000049(3)$ & $-0.000000018(10)$ & $0.00000003(3)$ \\
\hline 547.008 & $1.014546(2)$ & $0.00000093(3)$ & $0.000000505(13)$ & $-0.000000174(10)$ & $0.00000062(3)$ & $-0.00000001(2)$ & $0.000000012(4)$ \\
\hline 470.427 & $1.014647(4)$ & $0.00000122(7)$ & $0.000000605(15)$ & $-0.000000175(9)$ & $0.00000084(5)$ & $0.000000016(18)$ & $-0.00000002(4)$ \\
\hline 404.567 & $1.014778(3)$ & $0.00000186(8)$ & $0.00000090(4)$ & $-0.00000025(4)$ & $0.00000112(3)$ & $0.000000013(9)$ & $-0.00000008(5)$ \\
\hline 347.928 & $1.014919(3)$ & $0.00000238(12)$ & $0.00000118(3)$ & $-0.000000328(14)$ & $0.00000142(5)$ & $-0.00000002(2)$ & $-0.00000003(5)$ \\
\hline 299.218 & $1.015091(5)$ & $0.00000318(14)$ & $0.00000160(7)$ & $-0.00000027(4)$ & $0.00000203(3)$ & $0.00000001(7)$ & $-0.00000004(7)$ \\
\hline 257.327 & $1.015293(4)$ & $0.0000046(3)$ & $0.00000216(9)$ & $-0.00000046(7)$ & $0.00000274(9)$ & $-0.00000001(6)$ & $-0.00000011(18)$ \\
\hline 221.302 & $1.015505(5)$ & $0.00000543(8)$ & $0.00000304(8)$ & $-0.00000080(12)$ & $0.00000379(13)$ & $-0.00000019(4)$ & $0.00000016(18)$ \\
\hline 190.319 & $1.0157976(17)$ & $0.0000081(4)$ & $0.00000411(12)$ & $-0.00000104(10)$ & $0.0000058(3)$ & $-0.00000011(11)$ & $-0.0000000(2)$ \\
\hline 163.675 & $1.016100(9)$ & $0.0000110(7)$ & $0.00000546(15)$ & $-0.0000013(2)$ & $0.0000068(3)$ & $-0.0000002(3)$ & $-0.0000003(3)$ \\
\hline 140.76 & $1.016454(4)$ & $0.0000140(9)$ & $0.00000736(17)$ & $-0.00000210(17)$ & $0.0000089(3)$ & $0.0000002(1)$ & $-0.00000052(12)$ \\
\hline 121.054 & $1.016898(8)$ & $0.0000207(10)$ & $0.0000099(4)$ & $-0.0000031(5)$ & $0.0000136(6)$ & $0.0000002(4)$ & $0.0000001(8)$ \\
\hline 104.106 & $1.017433(16)$ & $0.0000300(18)$ & $0.0000138(6)$ & $-0.0000043(10)$ & $0.0000178(7)$ & $0.0000000(3)$ & $0.0000006(4)$ \\
\hline 89.531 & $1.01798(2)$ & $0.0000358(14)$ & $0.0000170(7)$ & $-0.0000062(3)$ & $0.0000239(14)$ & $0.0000000(9)$ & $-0.0000000(6)$ \\
\hline 76.997 & $1.01866(2)$ & $0.000052(3)$ & $0.0000251(9)$ & $-0.0000089(10)$ & $0.0000315(15)$ & $-0.0000002(8)$ & $0.000001(1)$ \\
\hline 66.217 & $1.01941(3)$ & $0.000068(4)$ & $0.0000326(6)$ & $-0.0000112(9)$ & $0.0000425(18)$ & $-0.0000001(9)$ & $0.000000(1)$ \\
\hline
\end{tabular}




\begin{tabular}{|c|c|c|c|c|c|c|c|}
\hline 1 & 2 & 3 & 4 & 5 & 6 & 7 & 8 \\
\hline 56.947 & $1.020351(18)$ & $0.000100(2)$ & $0.000047(2)$ & $-0.000013(3)$ & $0.000071(3)$ & $0.0000026(14)$ & $0.000003(2)$ \\
\hline 48.974 & $1.02144(3)$ & $0.000147(4)$ & $0.000066(3)$ & $-0.000027(3)$ & $0.000077(3)$ & $-0.0000013(13)$ & $0.000005(4)$ \\
\hline 42.118 & $1.02273(4)$ & $0.000196(3)$ & $0.000087(3)$ & $-0.000032(5)$ & $0.000113(8)$ & $-0.0000010(16)$ & $-0.000003(6)$ \\
\hline 36.221 & $1.02411(4)$ & $0.000268(5)$ & $0.000111(3)$ & $-0.000048(5)$ & $0.000158(3)$ & $0.000006(2)$ & $0.000002(6)$ \\
\hline 31.15 & $1.02592(4)$ & $0.00039(3)$ & $0.000157(5)$ & $-0.000067(10)$ & $0.000211(9)$ & $0.000019(4)$ & $-0.000019(6)$ \\
\hline 26.789 & $1.02804(5)$ & $0.00051(3)$ & $0.000204(4)$ & $-0.000093(9)$ & $0.000290(4)$ & $-0.000008(7)$ & $0.000014(11)$ \\
\hline 23.039 & $1.03063(9)$ & $0.00078(4)$ & $0.0003110(19)$ & $-0.000171(15)$ & $0.000442(13)$ & $0.000019(9)$ & $0.000001(16)$ \\
\hline 19.813 & $1.03353(10)$ & $0.00112(4)$ & $0.000422(13)$ & $-0.000250(17)$ & $0.000645(17)$ & $0.000021(16)$ & $-0.00008(3)$ \\
\hline 17.04 & $1.0368(2)$ & $0.00138(10)$ & $0.00065(4)$ & $-0.00037(5)$ & $0.00114(17)$ & $-0.00008(3)$ & $0.00000(3)$ \\
\hline 14.654 & $1.04088(16)$ & $0.00214(8)$ & $0.00092(4)$ & $-0.00062(3)$ & $0.00158(8)$ & $-0.00003(6)$ & $0.00024(10)$ \\
\hline 12.602 & $1.0459(3)$ & $0.0035(4)$ & $0.00152(9)$ & $-0.00112(18)$ & $0.009(5)$ & $-0.0004(2)$ & $0.0004(3)$ \\
\hline 10.838 & $1.0540(7)$ & $0.0070(15)$ & $0.0064(16)$ & $-0.0049(15)$ & $0.0043(3)$ & $0.0006(3)$ & $-0.0006(3)$ \\
\hline 9.321 & $1.06049(18)$ & $0.0067(5)$ & $0.0068(4)$ & $-0.0046(4)$ & $0.0049(3)$ & $-0.0003(3)$ & $0.0000(2)$ \\
\hline 8.016 & $1.06663(13)$ & $0.0079(10)$ & $0.0075(5)$ & $-0.0052(6)$ & $0.0054(6)$ & $-0.0001(3)$ & $0.0001(2)$ \\
\hline 6.894 & $1.07394(13)$ & $0.0063(4)$ & $0.0064(3)$ & $-0.0032(3)$ & $0.0055(3)$ & $-0.0004(3)$ & $0.00016(14)$ \\
\hline 5.929 & $1.0816(2)$ & $0.0073(2)$ & $0.0078(6)$ & $-0.0035(4)$ & $0.0057(3)$ & $0.0002(3)$ & $-0.0004(3)$ \\
\hline 5.099 & $1.08927(15)$ & $0.0098(3)$ & $0.0090(3)$ & $-0.0041(3)$ & $0.0074(3)$ & $-0.00082(4)$ & $0.0007(3)$ \\
\hline 4.385 & $1.0992(2)$ & $0.0107(3)$ & $0.0117(6)$ & $-0.0050(3)$ & $0.0079(3)$ & $0.0002(5)$ & $-0.0000(4)$ \\
\hline 3.771 & $1.10983(12)$ & $0.0142(9)$ & $0.0147(3)$ & $-0.0063(4)$ & $0.0092(5)$ & $-0.0005(3)$ & $0.0003(2)$ \\
\hline 3.243 & $1.12050(19)$ & $0.0182(5)$ & $0.0187(8)$ & $-0.0067(4)$ & $0.0124(5)$ & $0.00029(17)$ & $0.0000(4)$ \\
\hline 2.789 & $1.1331(3)$ & $0.0219(7)$ & $0.0216(4)$ & $-0.0074(3)$ & $0.0159(3)$ & $0.0001(3)$ & $0.0002(3)$ \\
\hline
\end{tabular}


Table IV. The equation of state and the dimensionless elastic compliances of the $\mathrm{J} 2$-structure of the heptamers $(\mathrm{N}=36)$

\begin{tabular}{|c|c|c|c|c|c|c|c|}
\hline $\mathrm{p}^{*}$ & $\mathrm{v}^{*}$ & $S_{11}$ & $\mathrm{~S}_{22}$ & $\mathrm{~S}_{12}$ & $\mathrm{~S}_{66}$ & $\mathrm{~S}_{62}$ & $S_{16}$ \\
\hline 1 & 2 & 3 & 4 & 5 & 6 & 7 & 8 \\
\hline 1000 & $1.0003303(13)$ & $0.000000204(4)$ & $0.000000138(4)$ & $0.000000017(4)$ & $0.000000140(3)$ & $0.000000001(3)$ & $-0.000000004(4)$ \\
\hline 860 & $1.0004312(06)$ & $0.000000259(13)$ & $0.000000207(9)$ & $0.000000020(6)$ & $0.000000182(8)$ & $-0.000000002(1)$ & $0.000000012(3)$ \\
\hline 739.6 & $1.000532(2)$ & $0.000000354(17)$ & $0.000000250(3)$ & $0.000000027(4)$ & $0.000000270(9)$ & $0.000000008(9)$ & $0.000000010(12)$ \\
\hline 636.056 & $1.000532(2)$ & $0.000000438(12)$ & $0.000000342(7)$ & $0.000000027(5)$ & $0.00000033(2)$ & $0.000000018(4)$ & $0.000000007(9)$ \\
\hline 547.008 & $1.0006331(18)$ & $0.00000070(3)$ & $0.000000456(13)$ & $0.000000026(10)$ & $0.000000467(6)$ & $0.000000013(9)$ & $-0.000000007(13)$ \\
\hline 470.427 & $1.000734(3)$ & $0.00000086(4)$ & $0.000000604(13)$ & $0.000000041(10)$ & $0.000000684(16)$ & $-0.000000042(10)$ & $0.000000001(15)$ \\
\hline 404.567 & $1.000936(3)$ & $0.00000117(5)$ & $0.00000087(3)$ & $0.00000008(4)$ & $0.000000804(8)$ & $-0.00000003(3)$ & $-0.00000001(3)$ \\
\hline 347.928 & $1.0010370(16)$ & $0.00000156(4)$ & $0.00000120(3)$ & $0.00000007(2)$ & $0.00000120(4)$ & $0.000000017(16)$ & $0.00000005(3)$ \\
\hline 299.218 & $1.001239(9)$ & $0.00000207(6)$ & $0.00000172(8)$ & $0.00000014(4)$ & $0.00000151(5)$ & $-0.00000003(5)$ & $-0.00000009(3)$ \\
\hline 257.327 & $1.001441(4)$ & $0.00000303(10)$ & $0.00000221(4)$ & $0.00000019(7)$ & $0.00000213(6)$ & $-0.00000002(3)$ & $-0.00000012(8)$ \\
\hline 221.302 & $1.001643(6)$ & $0.00000381(14)$ & $0.00000280(6)$ & $0.00000017(6)$ & $0.00000301(3)$ & $-0.00000005(4)$ & $-0.00000003(8)$ \\
\hline 190.319 & $1.001946(6)$ & $0.00000552(18)$ & $0.00000406(10)$ & $0.00000041(10)$ & $0.00000392(7)$ & $-0.00000004(11)$ & $0.00000001(11)$ \\
\hline 163.675 & $1.002248(6)$ & $0.0000073(4)$ & $0.0000055(2)$ & $0.00000067(17)$ & $0.00000536(12)$ & $0.00000023(6)$ & $0.00000026(10)$ \\
\hline 140.76 & $1.002551(11)$ & $0.0000098(3)$ & $0.00000744(14)$ & $0.0000008(2)$ & $0.0000071(4)$ & $-0.00000015(18)$ & $-0.00000011(9)$ \\
\hline 121.05$)$ & $1.002955(9)$ & $0.0000129(7)$ & $0.0000093(5)$ & $0.0000004(5)$ & $0.00000954(16)$ & $0.00000020(16)$ & $0.00000009(12)$ \\
\hline 104.106 & $1.003561(16)$ & $0.0000177(5)$ & $0.0000133(5)$ & $0.0000013(4)$ & $0.0000134(4)$ & $-0.0000004(3)$ & $-0.0000006(4)$ \\
\hline 89.531 & $1.004066(11)$ & $0.0000233(12)$ & $0.0000172(5)$ & $0.0000007(5)$ & $0.0000195(7)$ & $-0.0000005(3)$ & $-0.0000001(3)$ \\
\hline 76.997 & $1.004672(13)$ & $0.0000337(6)$ & $0.0000250(6)$ & $0.0000039(3)$ & $0.0000247(2)$ & $-0.0000002(3)$ & $-0.0000017(7)$ \\
\hline 66.217 & $1.00548(3)$ & $0.0000435(14)$ & $0.0000313(11)$ & $0.0000033(11)$ & $0.0000361(16)$ & $0.0000000(3)$ & $0.0000003(10)$ \\
\hline
\end{tabular}




\begin{tabular}{|c|c|c|c|c|c|c|c|}
\hline 1 & 2 & 3 & 4 & 5 & 6 & 7 & 8 \\
\hline 56.947 & $1.00649(2)$ & $0.0000590(19)$ & $0.0000452(18)$ & $0.0000034(3)$ & $0.0000447(14)$ & $-0.0000012(6)$ & $-0.0000017(10)$ \\
\hline 48.974 & $1.007499(19)$ & $0.000082(3)$ & $0.0000627(11)$ & $0.000006(3)$ & $0.000061(3)$ & $-0.0000006(10)$ & $-0.0000011(18)$ \\
\hline 42.118 & $1.00871(2)$ & $0.000114(4)$ & $0.000084(2)$ & $0.0000079(17)$ & $0.000085(2)$ & $-0.000001(\mathrm{I})$ & $-0.000005(4)$ \\
\hline 36.221 & $1.01063(6)$ & $0.000150(7)$ & $0.000107(3)$ & $0.000011(6)$ & $0.000115(5)$ & $-0.000001(3)$ & $-0.000001(5)$ \\
\hline 31.15 & $1.01164(5)$ & $0.000204(6)$ & $0.000145(3)$ & $0.000009(4)$ & $0.000170(3)$ & $-0.000007(5)$ & $-0.000005(3)$ \\
\hline 26.789 & $1.01366(3)$ & $0.000273(8)$ & $0.000207(4)$ & $0.000015(4)$ & $0.000237(7)$ & $-0.000004(3)$ & $0.000002(12)$ \\
\hline 23.039 & $1.01568(10)$ & $0.00037(2)$ & $0.000256(9)$ & $0.000023(3)$ & $0.000291(9)$ & $-0.000005(8)$ & $-0.000015(9)$ \\
\hline 19.813 & $1.01870(10)$ & $0.00051(3)$ & $0.000391(13)$ & $0.000043(12)$ & $0.00045(3)$ & $-0.000010(9)$ & $0.000008(9)$ \\
\hline 17.04 & $1.02173(8)$ & $0.00070(4)$ & $0.00051(2)$ & $0.000051(17)$ & $0.000594(19)$ & $-0.000004(15)$ & $0.000001(12)$ \\
\hline 14.654 & $1.02577(9)$ & $0.00098(2)$ & $0.000719(17)$ & $0.00003(4)$ & $0.00081(3)$ & $0.000001(11)$ & $-0.00002(3)$ \\
\hline 12.602 & $1.02981(13)$ & $0.00149(8)$ & $0.00094(4)$ & $0.000129(7)$ & $0.00112(7)$ & $-0.00001(3)$ & $-0.00002(4)$ \\
\hline 10.838 & $1.03486(8)$ & $0.00211(14)$ & $0.00123(4)$ & $0.00010(6)$ & $0.00171(4)$ & $0.00001(2)$ & $-0.00002(7)$ \\
\hline 9.321 & $1.04193(18)$ & $0.00271(12)$ & $0.00189(7)$ & $-0.00001(9)$ & $0.00243(7)$ & $0.00008(8)$ & $-0.00008(7)$ \\
\hline 8.016 & $1.04899(13)$ & $0.00441(19)$ & $0.00226(8)$ & $0.00008(8)$ & $0.00416(15)$ & $0.00008(9)$ & $0.0000(2)$ \\
\hline 6.894 & $1.0742(3)$ & $0.0066(4)$ & $0.0066(4)$ & $-0.0035(4)$ & $0.0052(3)$ & $0.0003(2)$ & $-0.0001(3)$ \\
\hline 5.929 & $1.0813(3)$ & $0.0082(4)$ & $0.0080(4)$ & $-0.0041(4)$ & $0.00576(14)$ & $0.0000(2)$ & $-0.0003(2)$ \\
\hline 5.099 & $1.0904(2)$ & $0.0094(6)$ & $0.0086(6)$ & $-0.0039(4)$ & $0.0070(3)$ & $0.0004(3)$ & $0.0001(2)$ \\
\hline 4.385 & $1.09947(15)$ & $0.0113(3)$ & $0.0108(3)$ & $-0.0045(5)$ & $0.00818(14)$ & $-0.0001(3)$ & $0.00036(18)$ \\
\hline 3.771 & $1.10957(16)$ & $0.0149(6)$ & $0.0141(3)$ & $-0.0056(6)$ & $0.0096(2)$ & $0.0000(2)$ & $0.0000(2)$ \\
\hline 3.243 & $1.1207(3)$ & $0.0166(6)$ & $0.0174(13)$ & $-0.0069(7)$ & $0.0119(9)$ & $-0.0002(4)$ & $0.0004(3)$ \\
\hline 2.789 & $1.1328(4)$ & $0.0223(3)$ & $0.0241(6)$ & $-0.0086(8)$ & $0.0161(5)$ & $-0.0006(5)$ & $0.0002(3)$ \\
\hline
\end{tabular}




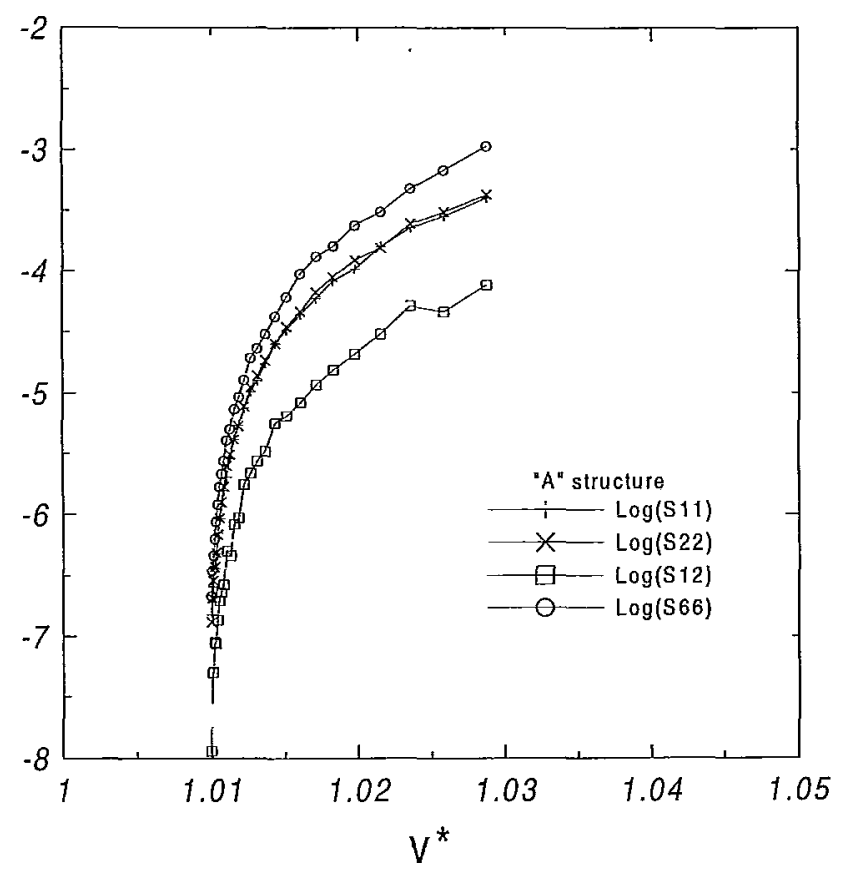

Fig. 6. The relative volume dependence of the elastic compliances of the A-structure of the heptamers

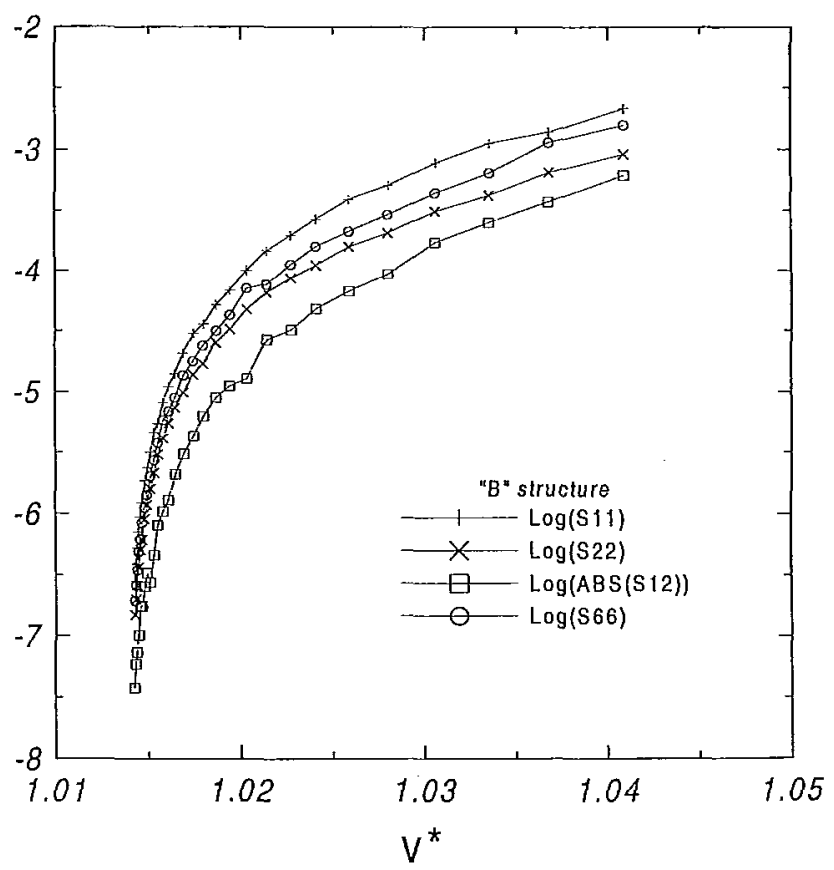

Fig. 7. The relative volume dependence of the elastic compliances of the B-structure of the heptamers 
Fig. 8. The relative volume dependence of the elastic compliances of the J2-structure of the heptamers

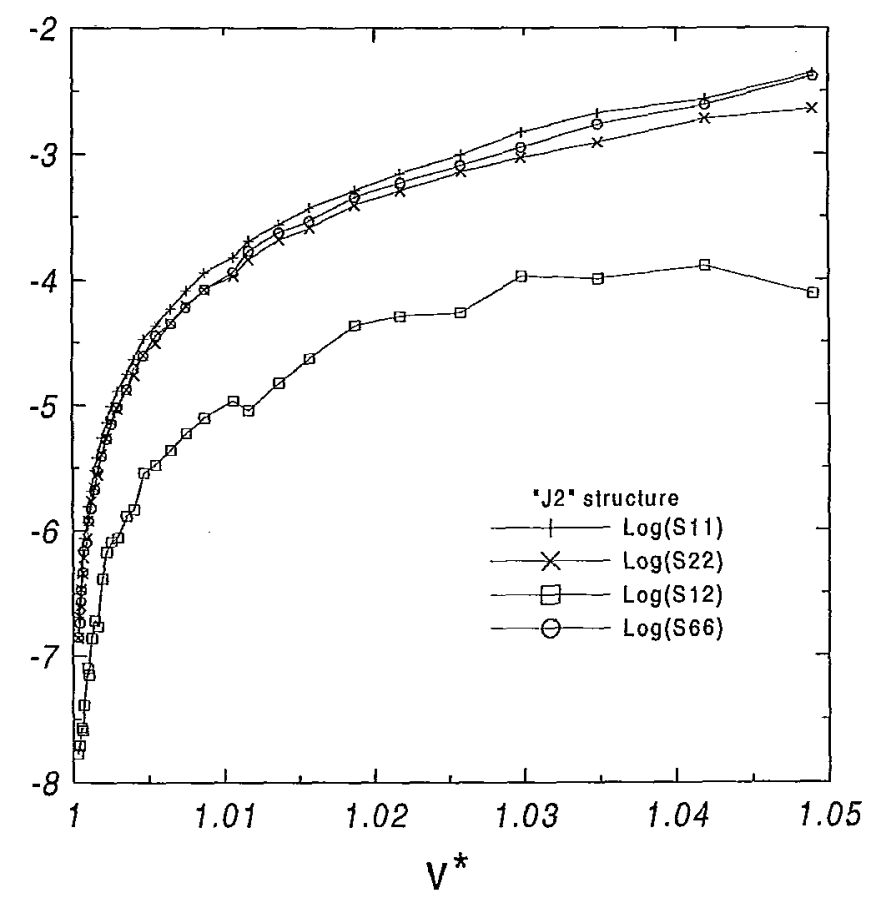

To test the dependence of the results on the system size (i.e. number of particles) a few series of runs were performed for various $N$ for the B-structure at a few pressures. The obtained elastic compliances are shown in Table I. In all tables and figures the Voigt notation is used. It can be seen that the results for systems of various sizes are within the obtained experimental errors. These results indicate that the elastic compliances of a perfect structure in the thermodynamic limit are well approximated (within a few percent accuracy) by even the smallest system studied. For this reason further studies have been done for systems consisted of $N=36$ heptamers.

The elastic compliances of the three dense crystalline structures (A, B, J2) of the heptamers are collected in Tables II-IV and their volume dependence is illustrated in Figs. 6-8. It can be seen that despite the anisotropy of the studied structures, what does not allow for exploiting symmetry to reduce experimental errors, the results are rather well convergent what means that the applied method can be effective for quite complicated intermolecular interactions.

Knowledge of the elastic compliances allows one to calculate all the elastic properties of the system, including the Poisson's ratio. As the dense structures of the heptamers are anisotropic, the Poisson's ratio can depend on the direction, in general. In Fig. 9 the dependencies of the Poisson's ratio on the relative volume are shown in the $x y$ and $y x$ directions. It is easy to notice that in the case of the B-structure the Poisson's ratios show a strong dependence on the direction and volume. In the denser structures A and J2 this dependencies are much weaker. 


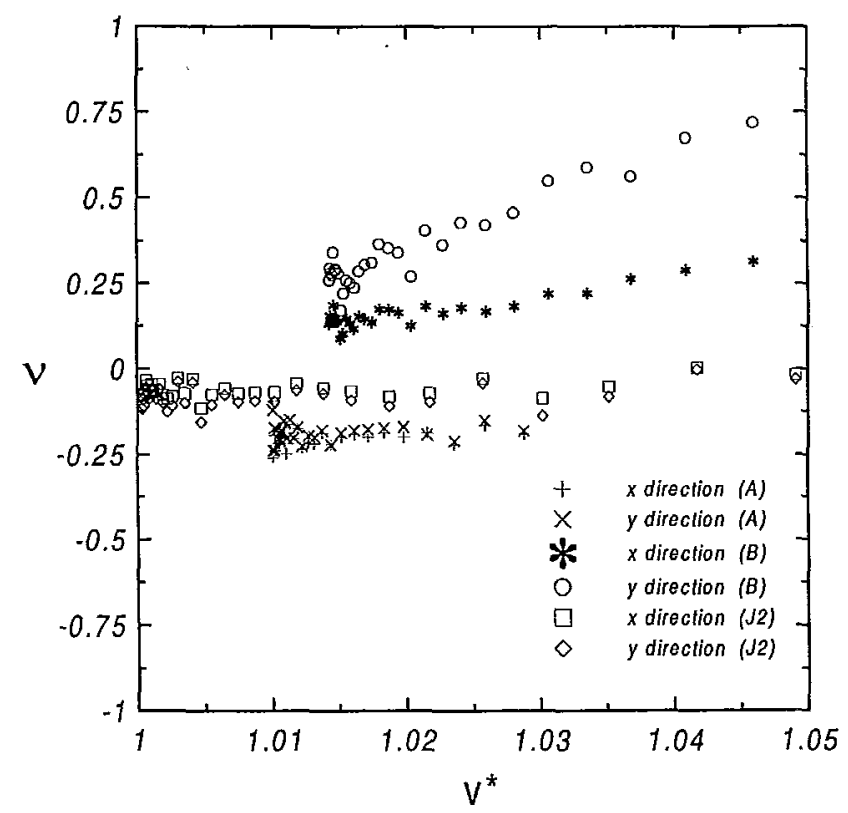

Fig. 9. The relative volume dependence of the Poisson's ratio for the structures A, B, and $\mathrm{J} 2$ of the heptamers

It should be stressed that the two densest structures of heptamers, A and J2, exhibit anomalous (negative) values of the Poisson's ratio, see Fig. 9.

\section{SUMMARY AND CONCLUSIONS}

Studies of the equation of state and structure of the heptamer system indicate that fluid freezes into a rotational phase of hexagonal lattice. Close to melting the atomic density distribution around the lattice sites is almost circular. At higher pressures, atomic patterns of 6-fold symmetry have been observed in this phase around each lattice site. This proves the existence of a strong translational-rotational coupling. Existence of a continuous phase transition between strongly hindered and almost free rotation is expected in this system in the vicinity of $p^{*}=2.8$. This is in a close analogy to the planar hard cyclic pentamers [10, 11],

At very high pressures the molecular rotation is frozen and the system forms a periodic lattice of lower symmetry. Three different crystalline structures were simulated at very high pressures. Thermodynamic stability ranges of these structures have not been determined. This is because standard simulations, like the present studies, are, in general, not able to distinguish between thermodynamically stable and metastable high-density structures. With no doubts, however, the simulated structures are metastable in certain ranges of density. This is sufficient to comupte their elastic compliances by using the strain-fluctuation method [12]. Despite the anisotropy of all the studied structures and the anisotropy of the intermolecular interactions of the heptamers, the results shown in Tables 2-4 indicate that the obtained method is sufficiently well convergent to give results within an accuracy of a few percent. This encourages one to apply this method to more complex systems. 
The data obtained in this paper can be used to extend possible tests of theoretical approximations for the elastic constants to anisotropic molecular systems.

It is worth to stress that some dense structures found in the heptamer system show negative values of the Poisson's ratio. This result may have interesting consequences for some granular systems.

\section{Acknowledgments}

We are grateful to Dr. A. C. Brańka for discussions. This work was supported by the (Polish) Committee for Scientific Research (KBN) within the grant 8T11F 01214. Part of the calculations was performed at the Poznań Supercomputer and Networking Center (PCSS).

\section{References}

[1] J.-P. Hansen, I. R. McDonald, Theory of Simple Liquids, Academic Press, London, 1986.

[2] M. P. Allen, G. T. Evans, D. Frenkel, B. Mulder, Hard Convex Body Fluids, in Advances in Chemical Physics, 86, 1-165 (1993). See also references therein.

[3] A. C. Brańka, K. W. Wojciechowski, Phys. Rev. Letters 50, 846-849 (1983).

[4] K. W. Wojciechowski, A. C. Brańka, D. Frenkel, Phys. Rev. Letters 64, 3168-3171 (1991); K. W. Wojciechowski, Phys. Rev. E46, 26-39 (1992); K. W. Wojciechowski, A. C. Brańka, D. Frenkel, Physica A196, 519-545 (1993).

[5] Yu. D. Burago, V. A. Zallgaller, Geometric Inequalities, Springer, Berlin 1988.

[6] R. Schneider, Convex bodies: The Brunn-Minkowski Theory, Vol. 44 of Encyclopedia of Mathematics and its Applications, Ed. G.-C. Rota, Cambridge, 1993.

[7] K. W. Wojciechowski, Physica A232, 723-736 (1996).

[8] K. W. Wojciechowski, J. Chem. Phys. 94, 4099-4100 (1991).

[9] S. Sachdev, D. R. Nelson, Phys. Rev. B32, 1480-1502 (1985).

[10] A. C. Brańka, K. W. Wojciechowski, Phys. Lett. A101, 349-353 (1984).

[11] A. C. Brańka, K. W. Wojciechowski, Mol. Phys. 72,941-953 (1991); A. C. Brańka, K. W. Wojciechowski, Mol. Phys. 78, 1513-1526 (1993).

[12] K. W. Wojciechowski, K. V. Tretiakov, Comput. Phys. Commun. 121-122, 528-530 (1999).

[13] B. L. Holian, O. E. Perçus, T. T. Warnock, P. A. Whitlock, Phys. Rev. E50, 1607-1615 (1994).

[14] M. Matsumoto and T. Nishimura, ACM Trans. Model. Comput. Simul. 8, 3-30 (1998).

[15] K. V. Tretiakov, K. W. Wojciechowski, Phys. Rev. E60, 7626 (1999); K. W. Wojciechowski, Comput. Meth. Sci. Technol. 5, 81-85 (1999).

[16] K. W. Wojciechowski, Mol. Phys. 61, 1247-1258 (1987); K. W. Wojciechowski and A. C. Brańka, Phys. Rev. A40, $7222-7225$ (1989).

[17] R. Lakes, Science 235, 1038 (1987).

[18] See, e.g., K. E. Evans, Endeavour, New Series 15, 170 (1991); R. Lakes, Adv. Mater. 5, 293 (1993); D. H. Boal, U. Seifert, J. C. Schillcock, Phys. Rev. E48, 4274 (1993); K. W. Wojciechowski, Mol. Phys. Reports 10,129 (1995); E. O. Martz, R. S. Lakes, J. B. Park, Cell. Polym. 15, 349 (1996); K. W. Wojciechowski and K. V. Tretiakov, Comput. Meth. Sci. Technol. 1, 25 (1996); D. Prali, R. Lakes, Int. J. Mechan. Sci. 39, 305 (1997); U. D. Larsen, O. Sigmund, S. Bouwstra, J. Macromech. Systems 6, 99 (1997).; P. S. Theocaris, G. E. Stavroulakis, P. D. Panagiotopoulos, Arch. Appl. Mechan. 67, 274 (1997); R. H. Baughman, J. M. Shacklette, A. A. Zakhidov, and S. Stafstroem, Nature 392, 362 (1998); K. W. Wojciechowski, Isotropic Systems of Negative Poisson Ratios, in Proceedings of the $2^{\text {rd }}$ Tohwa International Meeting, Eds. M. Tokuyama and I. Oppenheim (World Scientific, Singapore, 1998); G. Y. Wei and S. F. Edwards, Phys. Rev. E58, 6173 (1998); V. V. Novikov, K. W. Wojciechowski, Phys. Sol. State 41, 1970 (1999); R. H. Baughman, S. O. Dantas, S. Stafstrom, A. A. Zakhidov, T. B. Michell, D. H. E. Dubin, Science 288, 2018 (2000).

[19] K. W. Wojciechowski, A. C. Brańka, and M. Parrinello, Mol. Phys. 53, 1541-1546 (1984).

[20] M. Parrinello, A. Rahman, J. Chem. Phys. 76, 2662-2666 (1982). 